\title{
A High-Order Finite Element Method for the Linearised Euler Equations
}

\author{
K. Hamiche ${ }^{1), 2)}$, G. Gabard ${ }^{1)}$, H. Bériot ${ }^{2)}$ \\ 1) ISVR, University of Southampton, University Road, Southampton SO17 1BJ, United Kingdom. \\ kh2g12@soton.ac.uk, gabard@southampton.ac.uk \\ 2) Siemens PLM Software, Interleuvenlaan 68, 3001 Leuven, Belgium. \\ hadrien.beriot@siemens.com
}

\begin{abstract}
Summary
Sound propagation in complex non-uniform mean flows is an important feature of turbofan exhaust noise radiation. The Linearised Euler Equations are able to represent the strong shear layer refraction effects on the sound field, as well as multiple length scales. Frequency domain solvers are suitable for tonal noise and considered a way to avoid linear instabilities, which may occur with time domain solvers. However, the classical Finite Element Method suffers from dispersion error and high memory requirements. These shortcomings are particularly critical for high frequencies and for the Linearised Euler Equations, which involve up to five unknowns. In this paper, a high-order Finite Element Method is used to solve the Linearised Euler Equations in the frequency domain in order to overcome those issues. The model involves high-order polynomial shape functions, unstructured triangular meshes, numerical stabilisation and Perfectly Matched Layers. The acoustic radiation from a straight circular semi-infinite hard-wall duct with several mean flow configurations is computed. Comparisons with analytic solutions demonstrate the method accuracy. The acoustic and vorticity waves are well represented, as well as the refraction of the sound field across the jet shear layer. The high-order approach allows to use coarse meshes, while maintaining a sufficient accuracy. The benefits in terms of memory requirements are significant when compared to standard low-order Finite Element Method.
\end{abstract}

PACS no. 43.28.-g, 02.60.-x

\section{Introduction}

The International Civil Aviation Organisation directives are becoming more restrictive for aircraft noise. Accurate sound control tools are needed in order to respect these recommendations. In that context, efficient Computational AeroAcoustics prediction techniques are an alternative to costly large-scale measurements [1]. When dealing with turbofan exhaust noise, several physical aspects must be taken into consideration. Jet shear layers and boundary layers are responsible for sound refraction. In addition, vorticity and entropy waves may propagate and interact with acoustic waves. Numerical models must be able to represent both length scales.

Several mathematical models exist to represent those physical phenomena. The Linearised Potential Theory is developed for irrotational mean flows. Although it seems to hold with more general unsteady flows [2], it does not capture the vorticity/entropy

Received 30 January 2016, accepted 30 March 2016. waves. For complex mean flows where viscosity effects are not significant, the Linearised Euler Equations are a good compromise to represent acoustic propagation $[3,4]$. Their solutions support acoustic, vorticity, entropy waves [5], and their interactions. Linear instabilities which may pollute the solution are also supported $[6,7]$.

Several numerical methods have been proposed to solve the Linearised Euler Equations, either in time domain or in frequency domain [8]. Time domain solvers are preferred for their low-memory requirements and potentiality to parallelisation. Since a single simulation can provide results over large frequency range, they are also suited for broadband noise. The Discontinuous Galerkin Method has been applied for solving the Linearised Euler Equations [9]. However, issues related to impedance boundary conditions [10] and linear instabilities [11] are still under investigation.

By definition, frequency domain solvers are suitable for tonal noise. They are also an approach to avoid linear instabilities $[8,11]$. Since the stability criterion indicates that instabilities appear below a critical Strouhal number [6, 12], frequencies may be 
selected beyond the instability range. The Linearised Euler Equations have been solved in the frequency domain with structured and unstructured grids, using finite difference [13], discontinuous Galerkin [14] and linear Lagrangian finite element [15] methods. In the latter, the pressure gradients were neglected in the momentum equations which allowed to decouple the continuity equation and to solve only the momentum and energy equations. The main drawback of frequency domain approach is the high-memory requirements which constrain problems size and frequency.

In this paper, a high-order Finite Element Method ( $p$-FEM, with $p$ polynomial order) is presented for solving the axisymmetric Linearised Euler Equations in the frequency domain. The $p$-FEM approach allows to reduce the memory requirements by relying on high-order polynomials. In addition, a stabilised Galerkin formulation is used in order to suppress convection-related numerical instabilities [16].

This paper is structured as follows: the physical model is described in Section 2. The numerical method is then explained in Section 3. Finally, Section 4 describes the numerical results for sound radiation from a straight circular semi-infinite hard-wall duct with several mean flow velocity configurations.

\section{Physical Model}

\subsection{Linearised Euler Equations}

The Linearised Euler Equations represent sound wave propagation in non-uniform media for inviscid fluid and adiabatic process [17]. Each variable is expressed as the sum of a steady mean flow component (denoted by the subscript 0 ) and of a small amplitude unsteady perturbation (denoted by the superscript '). The equations are written for the conservative variables: the density $\rho$, the momentum $\rho \boldsymbol{u}$, and the modified pressure $p_{\mathrm{c}}=\left(p / p_{\infty}\right)^{1 / \gamma}$ introduced by Goldstein [18] (where $\boldsymbol{u}$ is the velocity vector, $p$ is the pressure, $p_{\infty}$ is a reference pressure, and $\gamma$ is the specific heats ratio). The variables vector is noted $\boldsymbol{Q}^{\prime}$.

The cylindrical coordinate system $(r, \theta, z)$ is convenient since mean flows and geometries are often axisymmetric. For time-harmonic perturbations, a Fourier decomposition of the solution yields:

$$
\boldsymbol{Q}^{\prime}(r, \theta, z, t)=\boldsymbol{q}^{\prime}(r, z) \mathrm{e}^{-\mathrm{j} m \theta} \mathrm{e}^{\mathrm{j} \omega t},
$$

where $t$ is the time, $m \in \mathbb{Z}$ is the azimuthal order and $\omega$ is the angular frequency.

The resulting Linearised Euler Equations read:

$$
\mathrm{j} \omega \boldsymbol{q}^{\prime}-\mathrm{j} \frac{m}{r} \boldsymbol{A}_{\theta} \boldsymbol{q}^{\prime}+\frac{1}{r} \boldsymbol{A}_{\mathrm{c}} \boldsymbol{q}^{\prime}+\frac{\partial \boldsymbol{A}_{z} \boldsymbol{q}^{\prime}}{\partial z}+\frac{1}{r} \frac{\partial r \boldsymbol{A}_{r} \boldsymbol{q}^{\prime}}{\partial r}=\mathbf{0},
$$

where the matrices $\boldsymbol{A}_{r}, \boldsymbol{A}_{\theta}$, and $\boldsymbol{A}_{z}$ contain the equations coefficients and characterise the fluxes in the $r$-, $\theta$ - and $z$-directions. The matrix $\boldsymbol{A}_{\mathrm{c}}$ also contains some equations coefficients. The matrices specific expressions are as follows:

$$
\begin{gathered}
\boldsymbol{A}_{\theta}=\left[\begin{array}{ccccc}
0 & 0 & 1 & 0 & 0 \\
0 & 0 & u_{0_{r}} & 0 & 0 \\
0 & 0 & 0 & 0 & \frac{\rho_{0} c_{0}^{2}}{p_{c_{0}}} \\
0 & 0 & u_{0_{z}} & 0 & 0 \\
0 & 0 & \frac{p_{c 0}}{\rho_{0}} & 0 & 0
\end{array}\right], \\
\boldsymbol{A}_{z}=\left[\begin{array}{ccccc}
0 & 0 & 0 & 0 \\
0 & 0 & 0 & 0 & -\frac{\rho_{0} c_{0}^{2}}{p_{\mathrm{c} 0}} \\
0 & 0 & 0 & 0 & 0 \\
0 & 0 & 0 & 0 \\
0 & 0 & 0 & 0 & 0
\end{array}\right], \\
\boldsymbol{A}_{\boldsymbol{c}}=\left[\begin{array}{ccccc}
0 & 0 & 0 & 1 & 0 \\
-u_{0_{z}} u_{0_{r}} & u_{0_{z}} & 0 & u_{0_{r}} & 0 \\
0 & 0 & u_{0_{z}} & 0 & 0 \\
-u_{0_{z}}^{2} & 0 & 0 & 2 u_{0_{z}} & \frac{\rho_{0} c_{0}^{2}}{p_{\mathrm{c}_{0}}} \\
-\frac{p_{\mathrm{c}_{0}}}{\rho_{0}} u_{0_{z}} & 0 & 0 & \frac{p_{c_{0}}}{\rho_{0}} & u_{0_{z}}
\end{array}\right], \\
0 \\
\left.\begin{array}{ccccc}
0 & 1 & 0 & 0 & 0 \\
-u_{0_{r}}^{2} & 2 u_{0_{r}} & 0 & 0 & \frac{\rho_{0} c_{0}^{2}}{p_{c_{0}}} \\
0 & 0 & u_{0_{r}} & 0 & 0 \\
-u_{0_{z}} u_{0_{r}} & u_{0_{z}} & 0 & u_{0_{r}} & 0 \\
-\frac{p_{c_{0}}}{\rho_{0}} u_{0_{r}} & \frac{p_{c_{0}}}{\rho_{0}} & 0 & 0 & u_{0_{r}}
\end{array}\right] .
\end{gathered}
$$

\subsection{Duct modes}

Acoustic sources can be described as duct modes, which form a complete basis for the duct incident wave [19]. A uniform mean flow is considered in the duct with axial velocity $u_{0}=u_{0_{z}}$. For cylindrical ducts, each modified pressure duct mode can be written:

$$
p_{\mathrm{c}_{m, n}}^{\prime}(r, \theta, z)=a_{m, n} U_{m, n}(r) \mathrm{e}^{-\mathrm{j} k_{z_{m}, n} z} \mathrm{e}^{-\mathrm{j} m \theta},
$$

where $n \in \mathbb{N}^{*}$ is the radial order, $a_{m, n}$ is the amplitude, $U_{m, n}$ is the radial shape function, and $k_{z_{m, n}}$ is the axial wavenumber. The resulting density and momentum components are obtained from the Linearised Euler Equations and have a form similar to Eq. 7.

The radial shape function for circular ducts is given by: $U_{m, n}(r)=J_{m}\left(k_{r_{m, n}} r\right)$, where $J_{m}$ is the $m^{\text {th }}$-order Bessel function of the first kind. The radial wavenumber $k_{r_{m, n}}=\alpha_{m, n} / r_{\mathrm{d}}$ depends on the $n^{\text {th }}$ zero $\alpha_{m, n}$ of the characteristic equation and on the duct outer radius $r_{\mathrm{d}}$. The characteristic equation is: $J_{m}^{\prime}\left(\alpha_{m, n}\right)=0$, where the prime denotes the derivative with respect to the argument.

\section{High-order finite element method}

\subsection{Formulation}

The physical problem defining the Linearised Euler Equations in Eq. 2 is transformed into an equivalent 
integral Galerkin formulation [20]:

$$
\begin{gathered}
\int_{\Omega} \mathrm{j} \omega r \boldsymbol{w}^{\mathrm{T}} \boldsymbol{q}^{\prime}-\mathrm{j} m \boldsymbol{w}^{\mathrm{T}} \boldsymbol{A}_{\theta} \boldsymbol{q}^{\prime}+\boldsymbol{w}^{\mathrm{T}} \boldsymbol{A}_{\mathrm{c}} \boldsymbol{q}^{\prime} \\
-r \frac{\partial \boldsymbol{w}^{\mathrm{T}}}{\partial z} \boldsymbol{A}_{z} \boldsymbol{q}^{\prime}-r \frac{\partial \boldsymbol{w}^{\mathrm{T}}}{\partial r} \boldsymbol{A}_{r} \boldsymbol{q}^{\prime} \mathrm{d} z \mathrm{~d} r \\
=-\int_{\Gamma} r \boldsymbol{w}^{\mathrm{T}}\left(n_{z} \boldsymbol{A}_{z}+n_{r} \boldsymbol{A}_{r}\right) \boldsymbol{q}^{\prime} \mathrm{d} \Gamma
\end{gathered}
$$

where $\Omega$ is the computational domain, $\boldsymbol{w}$ is the test function, $\left(n_{z}, n_{r}\right)$ are the components of the unit normal vector $\boldsymbol{n}$ to the boundary $\Gamma$. The superscript ${ }^{\mathrm{T}}$ denotes the Hermitian transpose. The matrix $\boldsymbol{F}=$ $n_{z} \boldsymbol{A}_{z}+n_{r} \boldsymbol{A}_{r}$ characterises the fluxes along $\boldsymbol{n}$, which points toward the domain exterior.

The method of characteristics provides a framework to formulate the boundary conditions for hyperbolic problems [21, 22]. The flux matrix $\boldsymbol{F}$ is diagonalised like: $\boldsymbol{F}=\boldsymbol{W} \boldsymbol{\Lambda} \boldsymbol{W}^{-1}$, where $\boldsymbol{\Lambda}$ is the eigenvalues diagonal matrix and $\boldsymbol{W}$ is the eigenvectors matrix. The eigenvectors define the amplitude vector $\hat{\boldsymbol{q}}$ of the characteristic waves travelling along $\boldsymbol{n}: \hat{\boldsymbol{q}}=\boldsymbol{W}^{-1} \boldsymbol{q}$. The eigenvalues represent each eigensolution phase velocity $\left(\lambda_{1}=\lambda_{2}=\lambda_{3}=\boldsymbol{u}_{0} \cdot \boldsymbol{n}, \lambda_{4}=\boldsymbol{u}_{0} \cdot \boldsymbol{n}-c_{0}\right.$, $\left.\lambda_{5}=\boldsymbol{u}_{0} \cdot \boldsymbol{n}+c_{0}\right)$, and indicate whether the characteristics are incoming or outgoing. Only the incoming waves must be imposed on a boundary [21]. For subsonic mean flows, $\lambda_{4}$ is strictly negative (incoming acoustic wave) and $\lambda_{5}$ is strictly positive (outgoing acoustic wave). The sign of $\boldsymbol{u}_{0} \cdot \boldsymbol{n}$ indicates whether the vorticity and entropy waves enter or leave the domain.

In practice, the flux vector $\boldsymbol{F} \boldsymbol{q}^{\prime}$ can be decomposed like: $\boldsymbol{F} \boldsymbol{q}^{\prime}=\boldsymbol{W} \boldsymbol{\Lambda} \boldsymbol{R} \boldsymbol{W}^{-1} \boldsymbol{q}^{\prime}+\hat{\boldsymbol{s}}$, where $\boldsymbol{R}$ is a reflection matrix and $\hat{s}$ is a source term. The boundary integral in Eq. 8 is rewritten like:

$$
\begin{aligned}
\int_{\Gamma} r \boldsymbol{w}^{\mathrm{T}} \boldsymbol{F} \boldsymbol{q}^{\prime} \mathrm{d} \Gamma \\
\quad=\int_{\Gamma} r \boldsymbol{w}^{\mathrm{T}} \boldsymbol{W} \boldsymbol{\Lambda} \boldsymbol{R} \boldsymbol{W}^{-1} \boldsymbol{q}^{\prime} \mathrm{d} \Gamma+\int_{\Gamma} r \boldsymbol{w}^{\mathrm{T}} \hat{\boldsymbol{s}} \mathrm{d} \Gamma .
\end{aligned}
$$

Within that scheme, the boundary conditions are imposed. Assuming hermetic acoustically rigid duct walls, the waves are completely reflected and no flow is transmitted through the wall. Slip hard-wall boundary conditions are applied with the normal velocity: $\boldsymbol{u}^{\prime} \cdot \boldsymbol{n}=0$ and $\boldsymbol{u}_{0} \cdot \boldsymbol{n}=0$.

Since the problem is axisymmetric, parity conditions constrain the variables continuity along the axis at $r=0$. For a cylindrical problem, the conditions are [23]:

$$
\begin{array}{cl}
u_{r}^{\prime}=u_{\theta}^{\prime}=0 & , \text { if } m=0, \\
\rho^{\prime}=0 ; u_{r}^{\prime}= \pm \mathrm{j} u_{\theta}^{\prime} ; u_{z}^{\prime}=0 ; p^{\prime}=0, & \text { if } m= \pm 1, \\
\rho^{\prime}=0 ; u_{r}^{\prime}=u_{\theta}^{\prime}=u_{z}^{\prime}=0 ; p^{\prime}=0, & \text { if }|m|>1 .
\end{array}
$$

\subsection{Finite element method}

In the finite element method, the continuous domain $\Omega$ is discretised into a finite number $n_{\mathrm{e}}$ of nonoverlapping linear elements $\Omega_{i}$. The solution vector $\boldsymbol{q}_{i}^{\prime}$ on each element splits up in terms of shape functions $S_{i}^{(j)}$ and of degrees of freedom $\boldsymbol{q}_{i}^{(j)}$ such that: $\boldsymbol{q}_{i}^{\prime}(\boldsymbol{x})=\sum_{j=1}^{n_{\mathrm{sf}}} \boldsymbol{q}_{i}^{(j)} S_{i}^{(j)}(\boldsymbol{x})$, where $\boldsymbol{x}$ is the position vector and $n_{\mathrm{sf}}$ is the number of shape functions. The test functions $\boldsymbol{w}$ use the same approximation basis.

Each element integral is evaluated by means of numerical quadrature [24], with accuracy order corresponding at least to the highest polynomial order in the integrand. For a single angular frequency $\omega$, the elementary integrals are assembled to produce a discrete global system of the form: $\boldsymbol{K} \boldsymbol{d}=\boldsymbol{f}$, where $\boldsymbol{K}$ is a square, sparse, complex matrix of size $n_{\text {dof }} \times n_{\text {dof }}$. The number of degrees of freedom $n_{\text {dof }}$ is equal to $5 n_{\mathrm{sf}}$, where the number 5 corresponds to the number of variables. $\boldsymbol{f}$ is the complex right-hand side vector. The linear system is solved with the multifrontal massively parallel sparse direct solver MUMPS [25], through the LU factorisation method which provides the unknown degrees of freedom.

The novelty in this work is that high-order shape functions are utilised to solve the Linearised Euler Equations. The standard FEM suffers from numerical dispersion and the so-called pollution effect [26]. The dispersion error increases with the frequency, which results in mesh refinement to obtain a reasonable accuracy. An alternative consists in using the $p$-FEM and incrementing the polynomial basis interpolation order. The enriched polynomial basis leads to lower resolution requirements and smaller number of degrees of freedom for a given problem, which results in lower memory usage $[27,28]$.

Although the high-order nodal Lagrange shape functions may be used, the high-order modal Lobatto shape functions are implemented in this work [29]. With modal shape functions, the $(p+1)$-degree polynomial basis is obtained as a correction of the $p$-degree polynomial basis. This hierarchic property makes modal shape functions a suitable choice for adaptivity. In addition, the Lobatto shape functions present good conditioning properties. The approximation basis contains not only vertex functions, but also edge and bubble shape functions. The vertex functions are linear and nodal. For $p \geq 2$, the basis is enriched with the edge functions: these functions are non-zero along a given edge and vanish along the others. For $p \geq 3$, the bubble functions are internal and vanish along the edges of the element.

Since they vanish along the reference element edges, the bubble shape functions are only local and have no connectivity with their neighbouring elements. Static condensation consists in expressing their corresponding degrees of freedom in terms of the remaining ones (vertex and edge degrees of freedom) within each element. These degrees of freedom are eliminated from 
the global system before assembly. Post-processing formulae permits to recover the solution internal degrees of freedom. This procedure translates into the reduction of the global system size, the conditioning improvement and the further decrease of the memory requirements [30]. Those effects become even more important for higher polynomial orders, since the number of bubble functions increases in $p^{2}$, making the use of static condensation crucial for high orders.

\subsection{Numerical stabilisation}

The finite element method experiences numerical instability for convection-dominated problems. The conventional Galerkin formulation introduced in Eq. 8 lacks stability for the Linearised Euler Equations [16]. This issue leads to the presence of spurious oscillations in the numerical solution. A stabilisation technique consists in adding artificial diffusion in each element:

$$
\begin{aligned}
\int_{\Omega} \mathrm{j} \omega r \boldsymbol{w}^{\mathrm{T}} \boldsymbol{q}^{\prime}-\mathrm{j} m \boldsymbol{w}^{\mathrm{T}} \boldsymbol{A}_{\theta} \boldsymbol{q}^{\prime}+\boldsymbol{w}^{\mathrm{T}} \boldsymbol{A}_{\mathrm{c}} \boldsymbol{q}^{\prime} \\
-r \frac{\partial \boldsymbol{w}^{\mathrm{T}}}{\partial z} \boldsymbol{A}_{z} \boldsymbol{q}^{\prime}-r \frac{\partial \boldsymbol{w}^{\mathrm{T}}}{\partial r} \boldsymbol{A}_{r} \boldsymbol{q}^{\prime} \mathrm{d} z \mathrm{~d} r \\
+\sum_{i=1}^{n_{\mathrm{e}}} \int_{\Omega_{i}} \mathfrak{D}(\boldsymbol{w})^{\mathrm{T}} \boldsymbol{\tau}_{i} \mathfrak{L}\left(\boldsymbol{q}^{\prime}\right) \mathrm{d} \Omega_{i} \\
=-\int_{\Gamma} r \boldsymbol{w}^{\mathrm{T}}\left(n_{z} \boldsymbol{A}_{z}+n_{r} \boldsymbol{A}_{r}\right) \boldsymbol{q}^{\prime} \mathrm{d} \Gamma,
\end{aligned}
$$

where $\mathfrak{D}(\boldsymbol{w})$ is a stabilisation operator, $\boldsymbol{\tau}_{i}$ is a stabilisation parameter and $\mathfrak{L}\left(\boldsymbol{q}^{\prime}\right)$ is the differential operator in the left-hand side of Eq. 2 [31].

Several stabilisation operators have been used in the literature [32]. In the following, the Galerkin/Least-Squares (GLS) technique is applied with:

$$
\begin{aligned}
\mathfrak{D}(\boldsymbol{w})=\mathrm{j} \omega \boldsymbol{w}-\mathrm{j} \frac{m}{r} \boldsymbol{A}_{\theta}^{\mathrm{T}} \boldsymbol{w}+\frac{1}{r} \boldsymbol{A}_{\mathrm{c}}^{\mathrm{T}} \boldsymbol{w} & \\
& +\frac{\partial \boldsymbol{A}_{z}^{\mathrm{T}} \boldsymbol{w}}{\partial z}+\frac{1}{r} \frac{\partial r \boldsymbol{A}_{r}^{\mathrm{T}} \boldsymbol{w}}{\partial r} .
\end{aligned}
$$

The matrices Hermitian transposes are used, providing better results, as observed for solving the Euler equations [33] and the Linearised Euler Equations [14]. The stabilisation parameter given by Rao and Morris is used [14]: $\boldsymbol{\tau}_{i}=\max \left(\alpha h_{i, l} / \rho_{l}\right) \boldsymbol{I}$, where $\alpha$ is a stabilisation coefficient, $h_{i, l}$ is the $i^{\text {th }}$-element size in the $l^{\text {th }}$-direction $(z$ or $r), \rho_{l}$ is the spectral radius of the matrix $\boldsymbol{A}_{l}$ and $\boldsymbol{I}$ is the identity matrix. In the simulations, $\alpha$ is chosen equal to $1 /(2 p)$ in order to take the high-order shape functions into consideration [16].

\subsection{Perfectly matched layer}

The physical domain truncation requires suitable nonreflecting boundary conditions to absorb outgoing waves. The Perfectly Matched Layer (PML) is a common technique based on the construction of an extra damping zone around the computational domain [34]. In that damping zone, the real physical coordinates are transformed into the complex plane:

$$
\tilde{z}(z)=z+\frac{f_{z}(z)}{\mathrm{j} k_{0}},
$$

where $\tilde{z}$ is the modified coordinate, $f_{z}$ is a stretching function and $k_{0}=\omega / c_{0}$ is the standard acoustic wavenumber. The logarithm function proposed by Bermúdez et al. is used [35]:

$$
f_{z}(z)=-n_{z} \ln \left(1-n_{z} \frac{z-z_{\mathrm{int}}}{d}\right),
$$

where $z_{\text {int }}$ denotes the interface coordinate and $d$ is the PML width. Analogous expressions are used for the absorption in the radial direction.

With flow, inverse upstream waves may propagate. These waves have positive group velocity but negative phase velocity, which complicates their absorption in the PML. The time dependency is transformed from $\mathrm{e}^{\mathrm{j} \omega t}$ to $\mathrm{e}^{\mathrm{j} \omega \tilde{t}}$ in the PML, allowing to translate the wavenumbers in the complex plane [36]:

$$
\tilde{t}(z, t)=t-\frac{\lambda_{z}}{\omega}\left(\tilde{z}(z)-z_{\text {int }}\right),
$$

where $\lambda_{z}$ is a correction coefficient. The most appropriate value of that parameter is shown to be [36]:

$$
\lambda_{z}=\frac{k_{0} M}{1-M^{2}},
$$

with $M=u_{0} / c_{0}$ the axial Mach number.

Applying the aforementioned space-time transformations, the Linearised Euler Equations in the PML read [15]:

$$
\begin{aligned}
\mathrm{j} \omega \boldsymbol{q}^{\prime}-\mathrm{j} \frac{m}{\tilde{r}} \boldsymbol{A}_{\theta} \boldsymbol{q}^{\prime}+\frac{1}{\tilde{r}} \boldsymbol{A}_{\mathrm{c}} \boldsymbol{q}^{\prime}+\mathrm{j} \lambda_{z} \boldsymbol{A}_{z} \boldsymbol{q}^{\prime} \\
+\frac{1}{\gamma_{z}} \frac{\partial \boldsymbol{A}_{z} \boldsymbol{q}^{\prime}}{\partial z}+\frac{1}{\tilde{r} \gamma_{r}} \frac{\partial \tilde{r} \boldsymbol{A}_{r} \boldsymbol{q}^{\prime}}{\partial r}=\mathbf{0},
\end{aligned}
$$

where $\tilde{r}$ is the modified coordinate in the $r$-direction, $\gamma_{z}=\mathrm{d} \tilde{z} / \mathrm{d} z$ and $\gamma_{r}=\mathrm{d} \tilde{r} / \mathrm{d} r$. Note that in the formulation a homogeneous Dirichlet boundary condition is applied at the PML outlet, such that $\boldsymbol{q}^{\prime}=\mathbf{0}$.

The PML can also be used to impose the incident wave. In practice, the PML equations are applied to the reflected field only $[13,15]: \boldsymbol{q}_{\mathrm{re}}^{\prime}=\boldsymbol{q}^{\prime}-\boldsymbol{q}_{\mathrm{in}}^{\prime}$, where $\boldsymbol{q}_{\text {in }}^{\prime}$ is the incident field and $\boldsymbol{q}_{\mathrm{re}}^{\prime}$ is the reflected field. The Linearised Euler Equations for incident wave injection in the PML are:

$$
\begin{aligned}
\mathrm{j} \omega \boldsymbol{q}^{\prime}-\mathrm{j} \frac{m}{\tilde{r}} \boldsymbol{A}_{\theta} \boldsymbol{q}^{\prime}+\frac{1}{\tilde{r}} \boldsymbol{A}_{\mathrm{c}} \boldsymbol{q}^{\prime}+\mathrm{j} \lambda_{z} \boldsymbol{A}_{z} \boldsymbol{q}^{\prime} \\
+\frac{1}{\gamma_{z}} \frac{\partial \boldsymbol{A}_{z} \boldsymbol{q}^{\prime}}{\partial z}+\frac{1}{\tilde{r} \gamma_{r}} \frac{\partial \tilde{r} \boldsymbol{A}_{r} \boldsymbol{q}^{\prime}}{\partial r} \\
=\mathrm{j} \omega \boldsymbol{q}_{\mathrm{in}}^{\prime}-\mathrm{j} \frac{m}{\tilde{r}} \boldsymbol{A}_{\theta} \boldsymbol{q}_{\mathrm{in}}^{\prime}+\frac{1}{\tilde{r}} \boldsymbol{A}_{\mathrm{c}} \boldsymbol{q}_{\mathrm{in}}^{\prime}+\mathrm{j} \lambda_{z} \boldsymbol{A}_{z} \boldsymbol{q}_{\mathrm{in}}^{\prime} \\
+\frac{1}{\gamma_{z}} \frac{\partial \boldsymbol{A}_{z} \boldsymbol{q}_{\mathrm{in}}^{\prime}}{\partial z}+\frac{1}{\tilde{r} \gamma_{r}} \frac{\partial \tilde{r} \boldsymbol{A}_{r} \boldsymbol{q}_{\mathrm{in}}^{\prime}}{\partial r} .
\end{aligned}
$$




\section{Application}

\subsection{Test case}

The numerical model is verified for the acoustic radiation from a straight circular semi-infinite duct [37]. The duct radius is $r_{\mathrm{d}}=1 \mathrm{~m}$. The duct wall is infinitely thin and hard-wall boundary conditions are applied on both sides. The mean flow velocity is axial, with Mach number $M_{\mathrm{d}}$ in the duct and $M_{\mathrm{o}}$ in the outer region. The mean flow density, speed of sound and specific heats ratio are uniform over the domain: $\rho_{0}=1.225 \mathrm{~kg} / \mathrm{m}^{3}, c_{0}=340.27 \mathrm{~m} / \mathrm{s}$ and $\gamma=1.4$. Figure 1 shows the test case configuration.

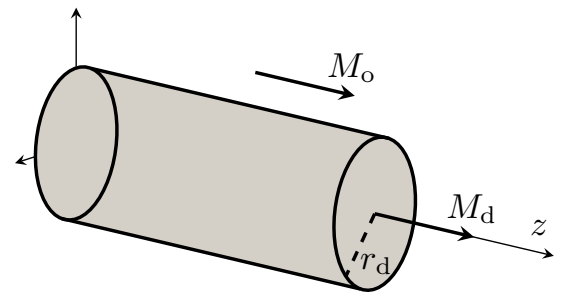

Figure 1. Straight circular duct geometry.

The computational domain extends from $z=0$ to $5 \mathrm{~m}$ and from $r=0$ to $2.5 \mathrm{~m}$. The duct exit plane is located at $z_{\mathrm{d}}=2.5 \mathrm{~m}$ (see Figure 2 ). Acoustic modes are injected inside the duct through a PML of length $d_{\mathrm{d}}$. In the outer region, the computational domain is surrounded by a PML of width $d_{\mathrm{o}}$. Axisymmetric boundary conditions are applied along the axis at $r=0$. The discretisation uses triangular elements, with a structured mesh inside the PML and an unstructured mesh elsewhere. The mesh is refined at the duct trailing edge in order to take the geometric singularity into account.

The numerical solution is compared to a reference solution [38], which is able to enforce the Kutta condition at the duct trailing edge. This analytic model reproduces the mean flow effects on the sound field, as it is seen in Sections 4.2.2 and 4.2.3. The solutions are compared on a 2 m-radius circle centred on the point $\left(z_{\mathrm{d}}, 0\right)$. The angle $\Phi$ along that circle is measured from the positive $z$-direction.

\subsection{Numerical results}

Three configurations are considered: the no-flow condition, the uniform flow condition and the nonuniform flow condition.

\subsubsection{No-flow condition}

The incoming acoustic wave is the time-harmonic plane wave $(m, n)=(0,1)$ with the angular frequency $\omega=6805 \mathrm{rad} / \mathrm{s}$, corresponding to the Helmholtz number $k_{0} r_{\mathrm{d}}=20$. The duct mode amplitude is $a_{0,1}=1$.
The characteristic mesh size is $0.4 \mathrm{~m}$ inside the duct and in the outer region, which corresponds to less than 1 element per wavelength. The element size at the duct trailing edge is $0.1 \mathrm{~mm}$. The PML are oneelement wide, both in the outer region and inside the duct $\left(d_{\mathrm{d}}=d_{\mathrm{o}}=0.4 \mathrm{~m}\right)$. Figure 2 shows the computational domain, the mesh and the control circle for comparison to the reference solution. The PML are shown with thicker lines, in blue for the outer region and in green for the duct mode injection. The duct wall is also represented with a thicker line.

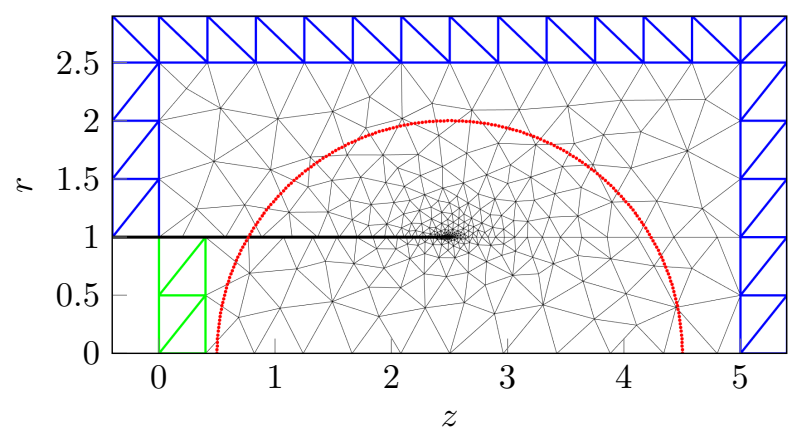

Figure 2. Computational domain, triangular mesh and control points. No-flow condition.

Figure 3 displays some numerical results with the variables fluctuations contours: the real parts of the modified pressure, the axial momentum and the radial momentum. These results are obtained with $p=9$. The solution shows the acoustic waves propagating inside the duct and radiating to the far field. The outgoing waves are properly absorbed inside the PML, with no noticeable reflections.

The numerical and analytic solutions are checked along the control circle. Figure 4 shows the Sound Pressure Level (SPL), calculated for a reference pressure $p_{\text {ref }}=2 \times 10^{-5} \mathrm{~Pa}$. The agreement is excellent. To further verify the numerical solution, the nodal error is integrated over the control circle. It is defined as the relative difference between the numerical and analytic solutions:

$$
E_{\chi}=\frac{\left\|\chi_{\mathrm{n}}-\chi_{\mathrm{a}}\right\|}{\left\|\chi_{\mathrm{a}}\right\|},
$$

where $\chi$ is the variable, the subscripts $n$ and a refer to the numerical and analytic solutions, and $\|\cdot\|$ is the 2-norm. For $p=9$, the numerical errors are: $E_{p_{\mathrm{c}}}=1.3 \%, E_{\rho u_{z}}=0.21 \%$ and $E_{\rho u_{r}}=4.5 \%$, which confirms the accuracy of the model. The maximum difference between the numerical and analytic SPL is $0.46 \mathrm{~dB}$.

Since the numerical model uses high-order polynomials, their impact is assessed in Figure 5. The errors along the control circle are plotted against the polynomial order. The low orders from $p=1$ to 4 are unable to solve the problem because the mesh is too 
Acta Acustica UnIted with Acustica Hamiche, Gabard, Bériot: A High-Order Finite Element Method for the Linearised Euler Equations

Vol. 01 (2016)
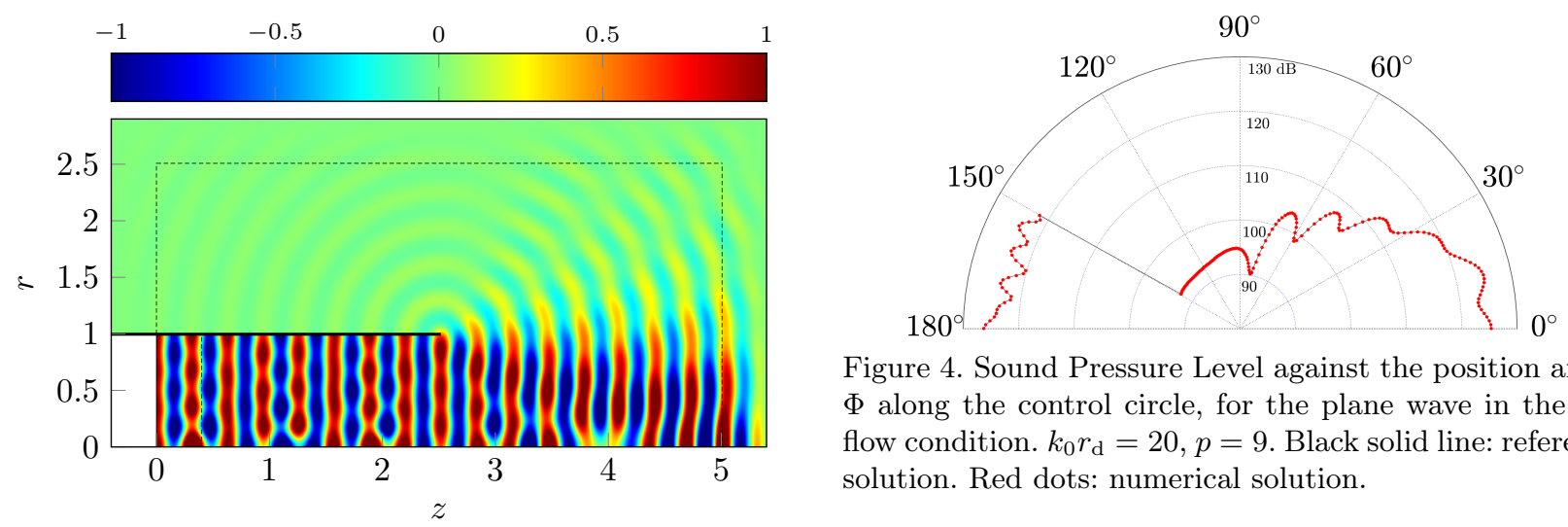

Figure 4. Sound Pressure Level against the position angle $\Phi$ along the control circle, for the plane wave in the noflow condition. $k_{0} r_{\mathrm{d}}=20, p=9$. Black solid line: reference solution. Red dots: numerical solution.
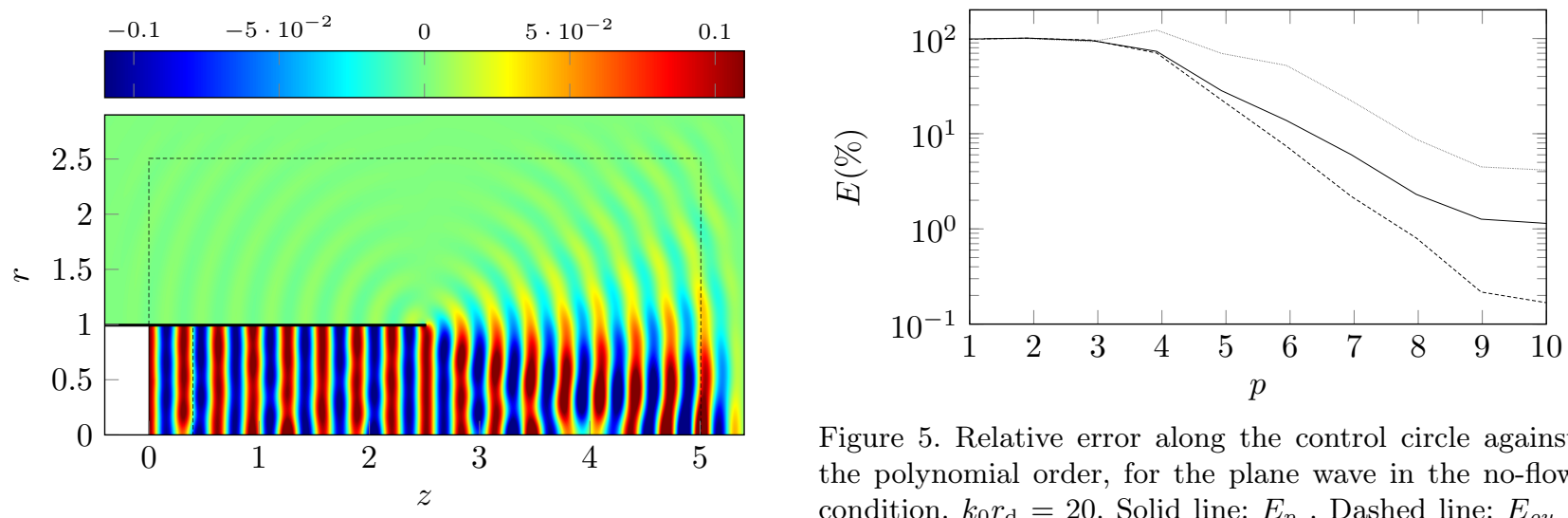

Figure 5. Relative error along the control circle against the polynomial order, for the plane wave in the no-flow condition. $k_{0} r_{\mathrm{d}}=20$. Solid line: $E_{p_{\mathrm{c}}}$. Dashed line: $E_{\rho u_{z}}$. Dotted line: $E_{\rho u_{r}}$.

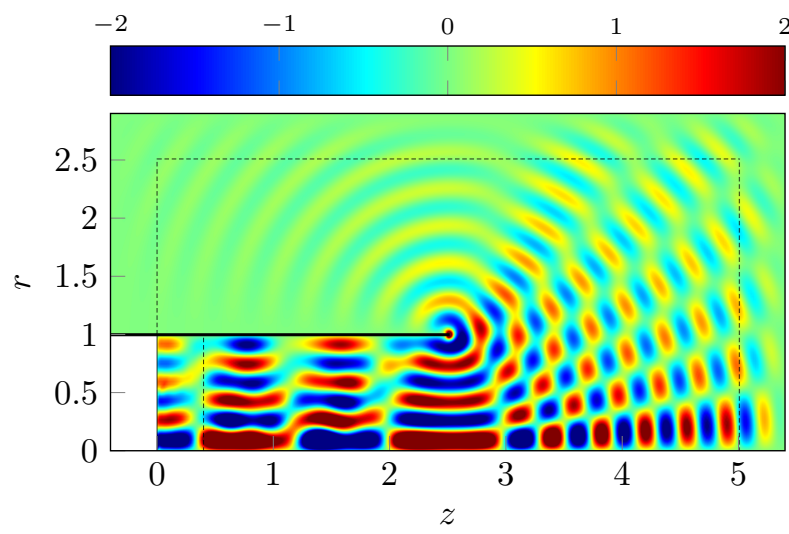

Figure 3. Contours of the variables perturbations, for the plane wave in the no-flow condition. $k_{0} r_{\mathrm{d}}=20, p=9$. Top: $\Re\left(p_{\mathrm{c}}\right)$. Middle: $\Re\left(\rho u_{z}\right)$. Bottom: $\Re\left(\rho u_{r}\right)$.

coarse. The solution convergence is observed since the numerical accuracy increases with the polynomial order, from $p=4$ to 9 . The errors stagnate for $p \geq 9$. This can be explained by the limited refinement at the duct trailing edge singularity. Moreover, the accuracy levels are different for the variables. The error on the $z$-momentum can be up to 20 times lower than the error on the $r$-momentum. This is due to the solution shape along the control circle: the radial momentum solution has more oscillations than the pressure and axial momentum solutions.

Concerning the performance, the memory usage to compute the numerical solution is $649 \mathrm{Mb}$ with $p=9$.

The number of degrees of freedom is 54,100 , for 818 triangular elements. For comparison, a simulation is ran for $p=2$ with a characteristic mesh size of $0.05 \mathrm{~m}$ inside the duct and in the outer region. This mesh corresponds to a number of 63,200 triangular elements. The numerical errors are: $E_{p_{\mathrm{c}}}=3.0 \%, E_{\rho u_{z}}=0.84 \%$ and $E_{\rho u_{r}}=11 \%$, which is still not as accurate as the simulation with $p=9$ and the coarse mesh. The memory usage is $4.5 \mathrm{~Gb}$, which is about 7 times higher than for the simulation with $p=9$. The number of degrees of freedom is 636,595 , i.e. almost 12 times the number found for the simulation with $p=9$.

\subsubsection{Uniform flow condition}

In the second configuration, the incoming duct mode $(5,1)$ propagates at the angular frequency $\omega=$ $2892 \mathrm{rad} / \mathrm{s}$. The Helmholtz number is $k_{0} r_{\mathrm{d}}=8.5$. The mean flow Mach number is uniform in the whole domain: $M_{\mathrm{d}}=M_{\mathrm{o}}=0.3$. The mean flow convects a vorticity wave from the duct trailing edge, which develops as a vortex sheet along the duct wake. The shortest acoustic wavelength is $0.57 \mathrm{~m}$ and the vorticity wavelength is $0.22 \mathrm{~m}$. In order to capture both effects, the mesh is refined accordingly. The characteristic mesh size is $0.4 \mathrm{~m}$ inside the duct and in the outer region. For the vorticity wave, the element size along the duct wake is $0.01 \mathrm{~m}$. Also in this case the PML are one-element wide, both in the outer region and inside the duct $\left(d_{\mathrm{d}}=d_{\mathrm{o}}=0.4 \mathrm{~m}\right)$. In addition 
to those located along the $2 \mathrm{~m}$-radius circle, control points are placed along the duct wall and its wake. Figure 6 shows the corresponding mesh.

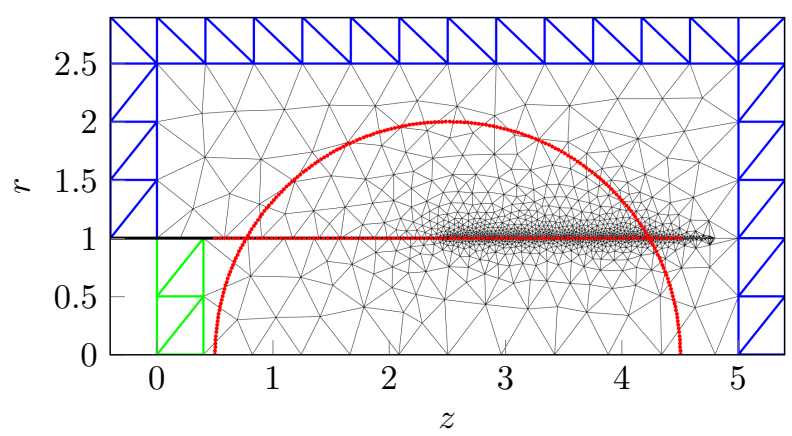

Figure 6. Computational domain, triangular mesh and control points. Uniform flow condition.

The variables perturbations contours are shown in the following pictures, with $p=9$. The pressure fluctuations are observed in the top picture in Figure 7: the noise propagates along the duct, radiates to the far field and is absorbed by the PML. The momentum fluctuations contours reveal the vortex sheet which develops from the duct trailing edge, as seen in the middle and bottom pictures in Figure 7 for the axial and for the radial components. The vortices are generated at the duct lip and are convected in the duct wake. The disparate acoustic and vorticity wavelengths are also observed. The axial momentum presents a very steep gradient across the vortex sheet, which requires a significant mesh refinement along the duct wake.

The Sound Pressure Level along the control circle shows the agreement between the numerical and analytic solutions. The sound directivity is seen in Figure 8 and the agreement between the solutions is very good. The nodal errors attest to the numerical model accuracy: $E_{p_{\mathrm{c}}}=0.32 \%, E_{\rho u_{z}}=2.2 \%$ and $E_{\rho u_{r}}=$ $1.3 \%$. The maximum difference between the numerical and analytic SPL is $0.3 \mathrm{~dB}$, for SPL $\geq 30 \mathrm{~dB}$.

The top picture in Figure 9 represents the radial momentum real part along the control circle, in linear scale. The axial momentum discontinuity at $\Phi \approx 150^{\circ}$ corresponds to the values on both sides of the duct wall, where the radial momentum is zero since the hard wall boundary condition is imposed. At $\Phi \approx 30^{\circ}$, the momentum components present a peak, which corresponds to the vorticity shedding. The bottom picture shows the radial momentum real part along the duct wall and its wake, also in linear scale. As expected, the normal velocity is zero along the duct wall $(z<2.5)$ and the oscillations along the vortex sheet are observed $(z>2.5)$. The solution continuity at $z=2.5$ is verified. The numerical solution matches the reference solution.

The numerical results converge to the analytic solution with the Kutta condition: the normal acoustic

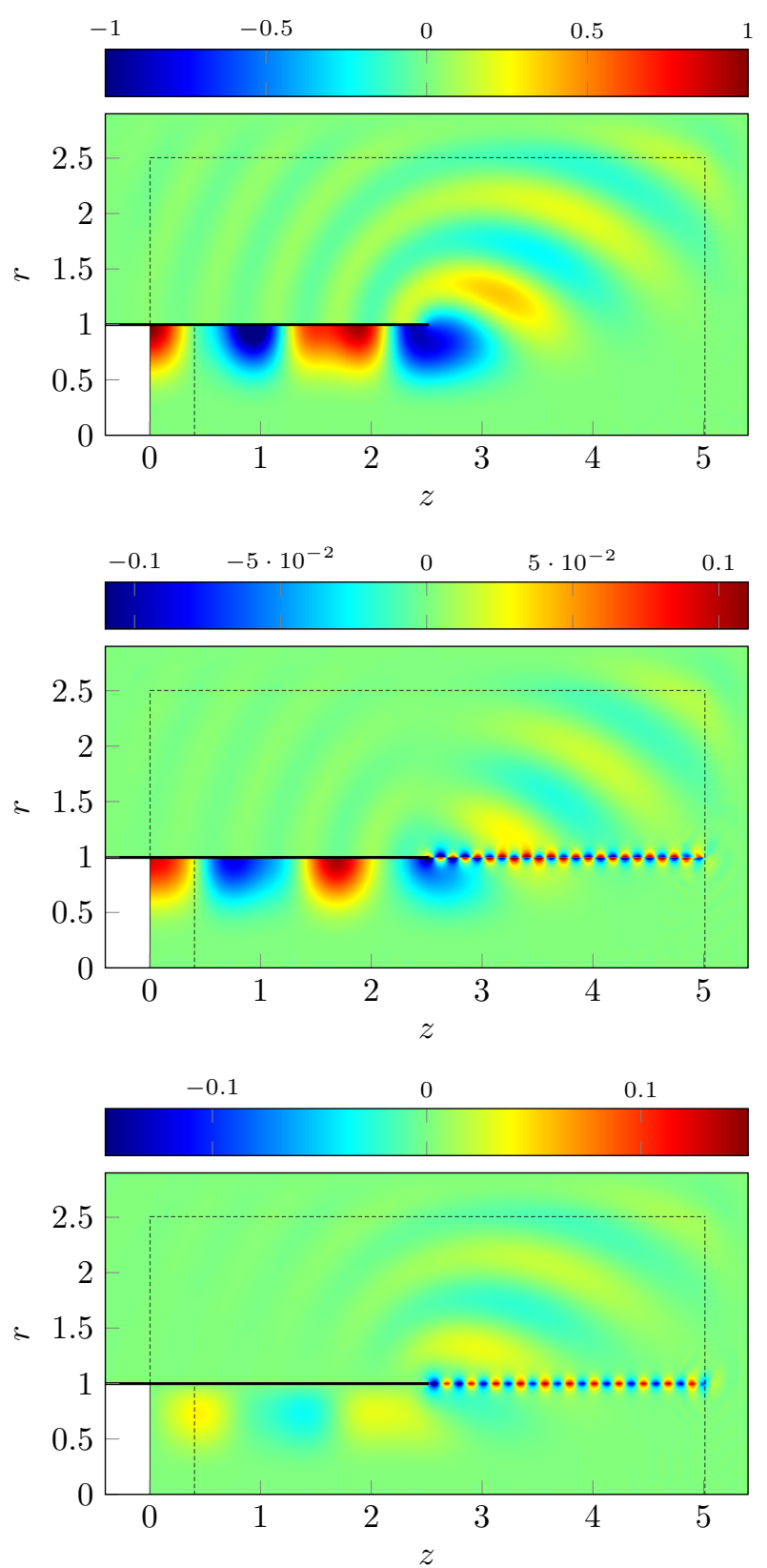

Figure 7. Contours of the variables perturbations, for the uniform flow condition. $k_{0} r_{\mathrm{d}}=8.5,(m, n)=(5,1), p=9$. Top: $\Re\left(p_{\mathrm{c}}\right)$. Middle: $\Re\left(\rho u_{z}\right)$. Bottom: $\Re\left(\rho u_{r}\right)$.

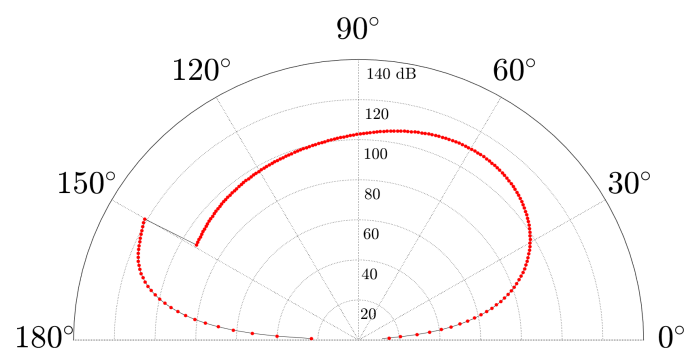

Figure 8. Sound Pressure Level against the position angle $\Phi$ along the control circle, for the uniform flow condition. $k_{0} r_{\mathrm{d}}=8.5,(m, n)=(5,1), p=9$. Black solid line: reference solution. Red dots: numerical solution. 
Acta Acustica UNITED with Acustica Hamiche, Gabard, Bériot: A High-Order Finite Element Method for the Linearised Euler Equations

Vol. 01 (2016)
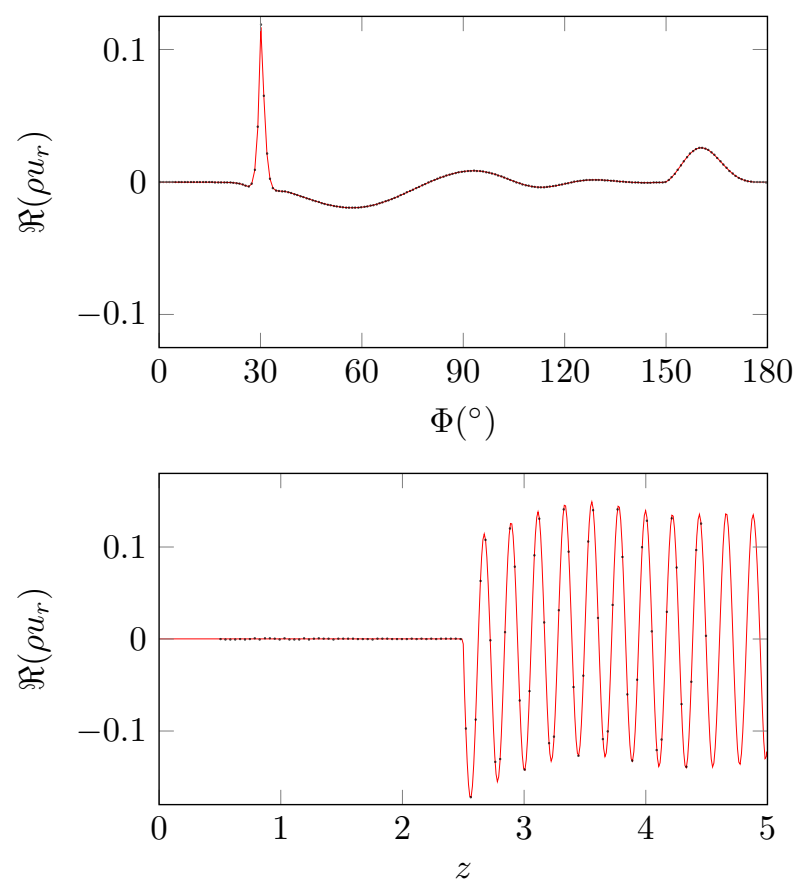

Figure 9. Radial momentum fluctuations, for the uniform flow condition. $k_{0} r_{\mathrm{d}}=8.5,(m, n)=(5,1), p=9$. Black dots: reference solution. Red solid line: numerical solution. Top: along the control circle. Bottom: along the duct wall and its wake.

velocity vanishes at the duct trailing edge and the solution is continuous at the trailing edge. The numerical solution naturally verifies that condition, which is implicitly imposed inside the numerical model: the Linearised Euler Equations support the pressure and velocity fields, and the shape functions continuity is ensured over the computational domain.

\subsubsection{Non-uniform flow condition}

The final configuration focuses on the non-uniform flow condition. Turbofan exhaust noise is characterised by mean flow shear layers with strong gradients. An analytic mean flow shear layer is built, in order to reproduce those features. The Mach number flow profile outside the duct is given by:

$$
M(r, z)=\bar{M}\left(1+\tanh \left(\frac{r_{\mathrm{d}}-|r|}{\zeta \delta(z)}\right)\right),
$$

where $\bar{M}=\left(M_{\mathrm{d}}+M_{\mathrm{o}}\right) / 2, \zeta=2 / 5 \cos ^{2}(\beta / 2)$ is a parameter to control the shear layer profile, $\beta$ is the spreading angle and $\delta$ is the shear layer thickness. The latter reads:

$$
\delta(z)=2\left(z-z_{\mathrm{d}}\right) \tan (\beta / 2) .
$$

The following parameters are used: $M_{\mathrm{d}}=0.5, M_{\mathrm{o}}=0$ and $\beta=20^{\circ}$. Within each finite element, the mean flow velocity profile is imposed directly on each Gauss point of the quadrature. Figure 10 shows the mean flow Mach number contours.

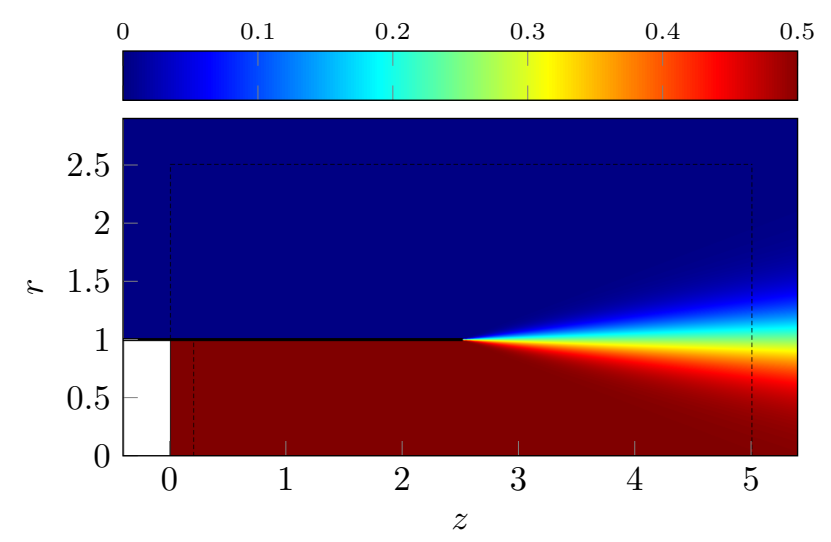

Figure 10. Contours of the mean flow Mach number, for the non-uniform flow condition. $M_{\mathrm{d}}=0.5, M_{\mathrm{o}}=0$.

The incoming acoustic wave is the duct mode $(10,1)$ with the angular frequency $\omega=5785 \mathrm{rad} / \mathrm{s}$. The Helmholtz number is $k_{0} r_{\mathrm{d}}=17$. The corresponding shortest acoustic wavelength is $0.18 \mathrm{~m}$, and is equal to the vorticity wavelength. The computational domain is discretised with the mesh depicted in Figure 11. The mesh size is $0.4 \mathrm{~m}$ in the outer region. Inside the duct, the mesh is refined with a characteristic size of $0.2 \mathrm{~m}$. The refinement along the duct wake is $0.01 \mathrm{~m}$, in order to capture the vorticity shedding from the duct trailing edge in the axial direction. The PML are one-element wide, with $d_{\mathrm{d}}=0.2 \mathrm{~m}$ and $d_{\mathrm{o}}=0.4 \mathrm{~m}$.

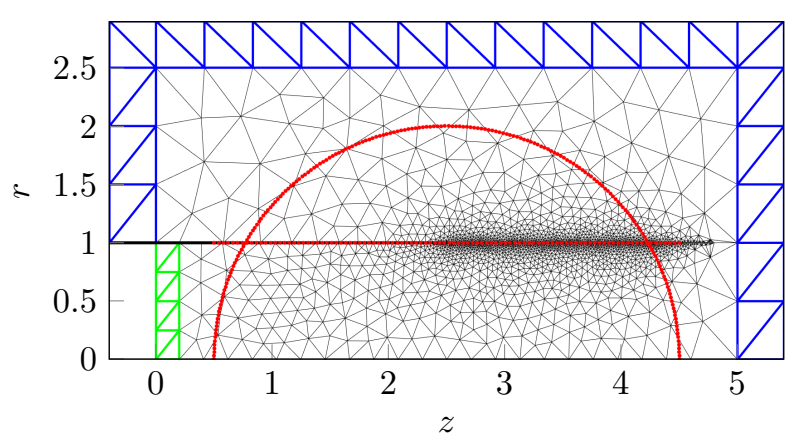

Figure 11. Computational domain, triangular mesh and control points. Non-uniform flow condition.

The perturbations contours are shown in Figure 12 for the modified pressure, the $z$-momentum and the $r$-momentum, with $p=8$. The sound propagation, refraction and radiation to the far field are visible. The shear layer is responsible for the generation of the hydrodynamic Kelvin-Helmholtz instability, which develops as a vorticity shedding along the duct wake and decays after a finite distance.

The Sound Pressure Level along the control circle is shown in Figure 13. The analytic solution available without vorticity shedding is also represented, as an element of reference. It shows that the numerical solution is very similar, and that the vortex sheet does not significantly impact the acoustic directivity. 

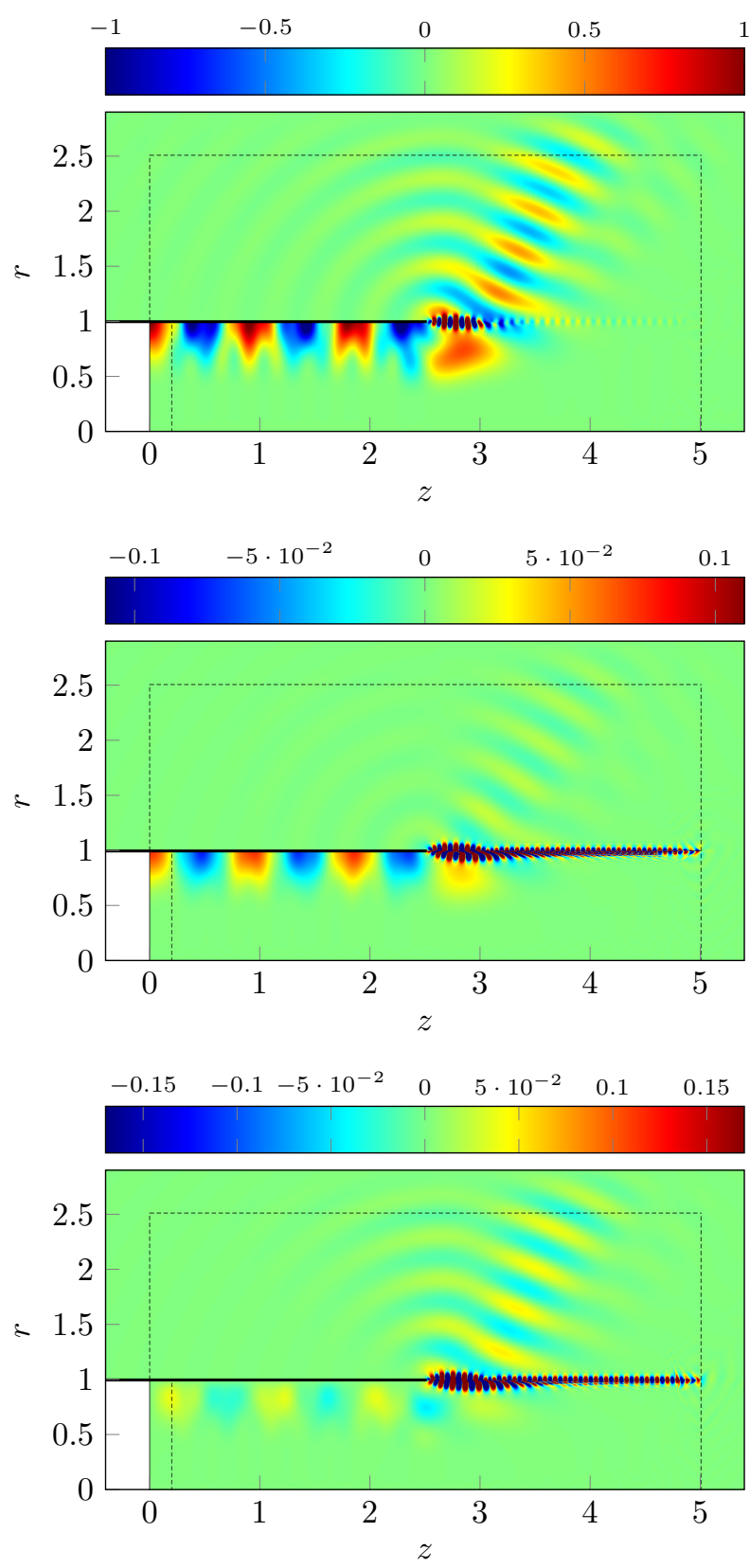

Figure 12. Contours of the variables perturbations, for the non-uniform flow condition. $k_{0} r_{\mathrm{d}}=17,(m, n)=(10,1)$, $p=8$. Top: $\Re\left(p_{\mathrm{c}}\right)$. Middle: $\Re\left(\rho u_{z}\right)$. Bottom: $\Re\left(\rho u_{r}\right)$.

Around the angle $\Phi=30^{\circ}$, a peak corresponding to the vortex sheet is observed. This peak is not visible on the reference solution, since the Kutta condition is not applied. The non-uniform mean flow is responsible for the wave refraction outside the duct.

The solution convergence is assessed in Figure 14 which displays the variables real parts along the duct wall and its wake, for $p=2,3,7$ and 8 . Along the duct wall, the solution has already converged with $p=2$ : the oscillations are visible on the pressure and $z$-momentum plots, while the normal velocity along the hard wall is verified to be zero on the $r$-momentum plot. The longest acoustic wavelength $0.55 \mathrm{~m}$ domi- nates there and the resolution with $p=2$ is already sufficient to solve it. The reference solution obtained with no vorticity shedding is identical to the numerical solution, which means that the solution along the duct wall is not affected in this test case. At the duct trailing edge, the Kelvin-Helmholtz instability wave is generated: it grows exponentially and then decreases $[6,7]$. This instability is not supported by the reference solution. Some vorticity wave with constant amplitude is still present on the momentum plots, but its effect on the pressure field is less significant. Those oscillations, which characterise the vorticity wavelength $0.18 \mathrm{~m}$, are not well represented by the orders $p<6$. The coupling between acoustic and vorticity waves is visible on the pressure plot. It is mainly limited to the instability range (from $z=2.5$ to 3 ), even though some oscillations of significantly lower amplitude are present outside that range. These oscillations seem to be of a similar nature as those observed in the uniform flow condition, which amplitude remains constant along the duct wake. The mathematical model does not take into consideration the non-linear effects and the viscosity which may attenuate these oscillations in real cases. With the classical linear Finite Element Method, a much finer mesh should be used in order to solve this problem. The high-order finite element method is able to reproduce the features of the pressure field but also the strong oscillations present in the momentum field.

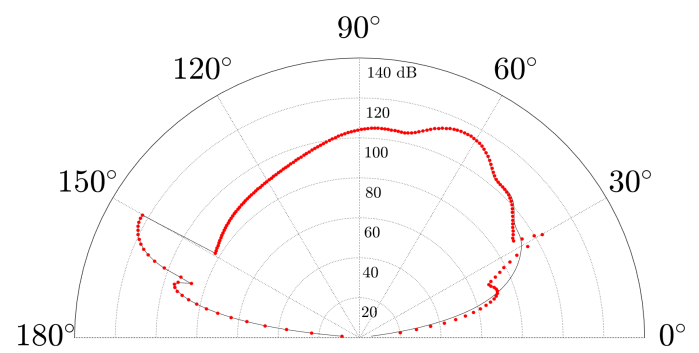

Figure 13. Sound Pressure Level against the position angle $\Phi$ along the control circle, for the non-uniform flow condition. $k_{0} r_{\mathrm{d}}=17,(m, n)=(10,1), p=8$. Black solid line: reference solution (no vorticity shedding). Red dots: numerical solution.

\section{Conclusion}

A novel frequency domain approach based on a highorder Finite Element Method is developed to solve the Linearised Euler Equations. As a verification, the acoustic radiation from a straight circular semiinfinite hard-wall duct is successfully computed for several mean flow conditions.

The comparison with an analytic solution shows that the numerical solutions accurately describe the sound propagation, as well as the vorticity waves 
Acta Acustica UNITED with Acustica Hamiche, Gabard, Bériot: A High-Order Finite Element Method for the Linearised Euler Equations

Vol. 01 (2016)
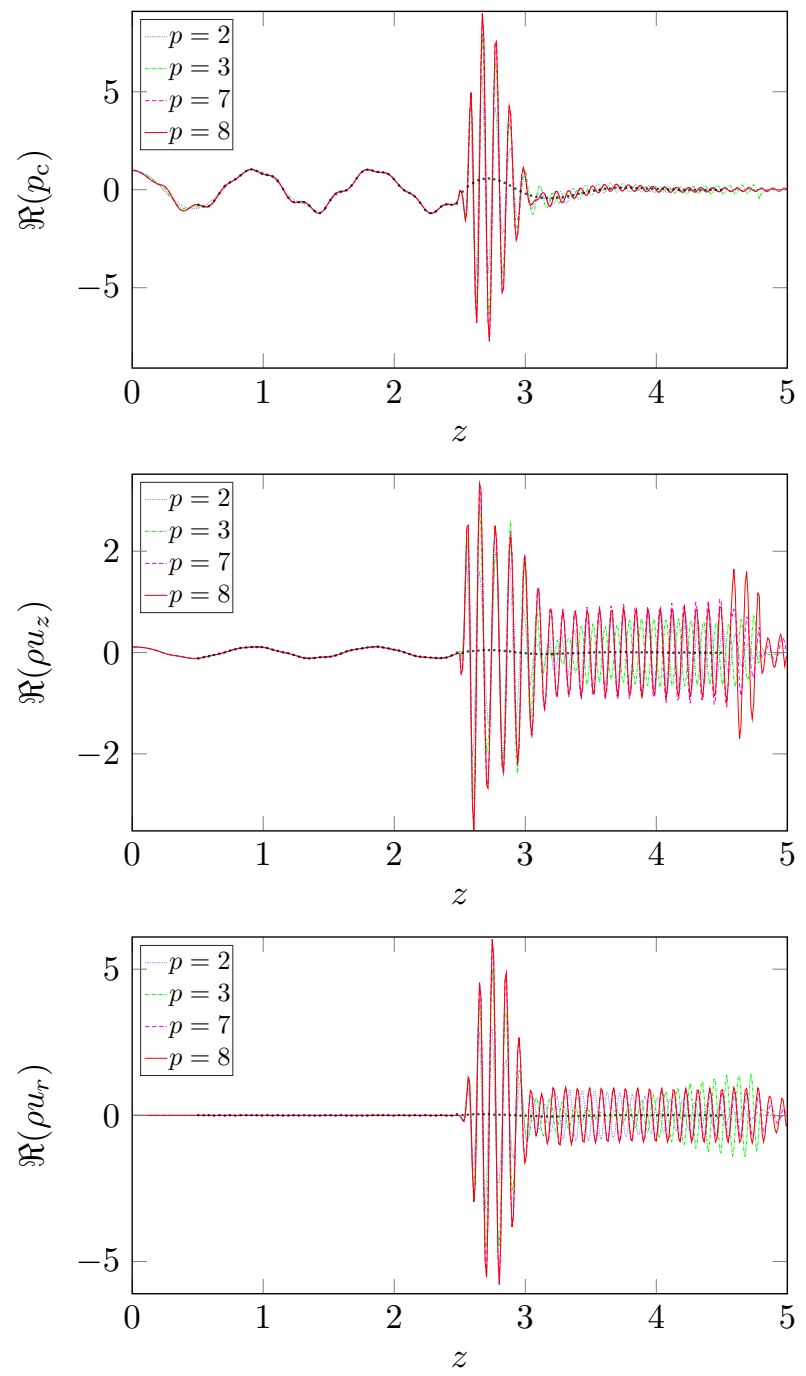

Figure 14. Variables fluctuations along the duct wall and its wake, for the non-uniform flow condition. $k_{0} r_{\mathrm{d}}=17$, $(m, n)=(10,1), p=2,3,7$ and 8 . Top: $\Re\left(p_{\mathrm{c}}\right)$. Middle: $\Re\left(\rho u_{z}\right)$. Bottom: $\Re\left(\rho u_{r}\right)$. Black dots: reference solution (no vorticity shedding).

and their interaction with the acoustic field. With a mean flow shear layer, the Kelvin-Helmholtz instability wave grows with the flow and then decays. A lower amplitude vorticity wave still propagates downstream of that instability. The refraction of the sound field through the shear layer is observed.

The high-order Finite Element Method is confirmed to be more efficient than the low-order Finite Element Method. The memory requirements are significantly reduced with the $p$-FEM, with a factor 7 for the no-flow condition test case. The convergence of the method is verified when $p$ is increased. Mesh refinement is however necessary to deal with local geometrical singularities and steep-gradient solutions. Local $p$-adaptivity may further improve the performance.

\section{Acknowledgement}

The authors gratefully acknowledge the financial support from the European Commission through the FP7-PEOPLE-ITN-2011 FlowAirS project, grant agreement 289352, coordinated by Y. Aurégan from the Laboratoire d'Acoustique de l'Université du Maine (France).

\section{References}

[1] R. J. Astley: Numerical Methods for Noise Propagation in Moving Flows, with Application to Turbofan Engines. Acoustical Science and Technology 30 (2009) 227-239.

[2] A. Pierce: Wave Equation for Sound in Fluids with Unsteady Inhomogeneous Flow. Journal of the Acoustical Society of America 87 (1990) 2292-2299.

[3] C. Bailly, D. Juvé: Numerical Solution of Acoustic Propagation Problems Using Linearized Euler Equations. AIAA Journal 38 (2000) 22-29.

[4] C. Bogey, C. Bailly, D. Juvé: Computation of Flow Noise Using Source Terms in Linearized Euler Equations. AIAA Journal 40 (2002) 235-243.

[5] C. K. W. Tam, J. C. Webb: Dispersion-RelationPreserving Finite Difference Schemes for Computational Acoustics. Journal of Computational Physics 107 (1993) 262-281.

[6] A. Michalke: On Spatially Growing Disturbances in an Inviscid Shear Layer. Journal of Fluid Mechanics 23 (1965) 521-544.

[7] P. G. Drazin: Introduction to Hydrodynamic Stability. Cambridge University Press, Cambridge, 2002.

[8] A. Angeloski, M. Discacciati, C. Legendre, G. Lielens, A. Huerta: Challenges for Time and Frequency Domain Aeroacoustic Solvers. $11^{\text {th }}$ World Congress on Computational Mechanics, Barcelona, 2014.

[9] R. D. R. Rinaldi, A. Iob, R. Arina: An Efficient Discontinuous Galerkin Method for Aeroacoustic Propagation. International Journal for Numerical Methods in Fluids 69 (2012) 1473-1495.

[10] G. Gabard, E. J. Brambley: A Full Discrete Dispersion Analysis of Time-Domain Simulations of Acoustic Liners with Flow. Journal of Computational Physics 273 (2014) 310-326.

[11] A. Agarwal, P. J. Morris, R. Mani: The Calculation of Sound Propagation in Non-Uniform Flows: Suppression of Instability Waves. AIAA Journal 42 (2004) 80-88.

[12] A. Michalke: Survey on Jet Instability Theory. Progress in Aerospace Sciences 21 (1984) 159-199.

[13] Y. Özyörük: Numerical Prediction of Aft Radiation of Turbofan Tones Through Exhaust Jets. Journal of Sound and Vibration 325 (2009) 122-144.

[14] P. Rao, P. J. Morris: Use of Finite Element Methods in Frequency Domain Aeroacoustics. AIAA Journal 44 (2006) 1643-1652.

[15] A. Iob, R. Arina, C. Schipani: Frequency-Domain Linearized Euler Model for Turbomachinery Noise Radiation Through Engine Exhaust. AIAA Journal 48 (2010) 848-858.

[16] J. Donea, A. Huerta: Finite Element Methods for Flow Problems. John Wiley \& Sons, Ltd, Chichester, 2003. 
[17] G. K. Batchelor: An Introduction to Fluid Dynamics. Cambridge University Press, Cambridge, 1967.

[18] M. E. Goldstein: An Exact Form of Lilley's Equation with a Velocity Quadrupole/Temperature Dipole Source Term. Journal of Fluid Mechanics 443 (2001) 231-236.

[19] S. W. Rienstra, A. Hirschberg: An Introduction to Acoustics. Eindhoven University of Technology, Eindhoven, 2013.

[20] O. C. Zinkiewicz, R. L. Taylor: The Finite Element Method, Volume 1, The Basis. ButterworthHeinemann, Oxford, 2000.

[21] R. L. Higdon: Initial-Boundary Value Problems for Linear Hyperbolic Systems. SIAM Review 28 (1986) 177-217.

[22] R. Hixon: Radiation and Wall Boundary Conditions for Computational Aeroacoustics: A Review. International Journal of Computational Fluid Dynamics 18 (2004) 523-531.

[23] H. R. Lewis, P. M. Bellan: Physical Constraints on the Coefficients of Fourier Expansions in Cylindrical Coordinates. Journal of Mathematical Physics 31 (1990) 2596-2596.

[24] D. A. Dunavant: High Degree Efficient Symmetrical Gaussian Quadrature Rules for the Triangle. International Journal for Numerical Methods in Engineering 21 (1985) 1129-1148.

[25] MUMPS Team: MUMPS 4.10.0: User's Guide. Tech. Rept. 2011.

[26] F. Ihlenburg, I. Babuška: Finite Element Solution of the Helmholtz Equation with High Wave Number, Part I: The $h$-Version of the FEM. Computers \& Mathematics with Applications 30 (1995) 9-37.

[27] H. Bériot, G. Gabard, E. Perrey-Debain: Analysis of High-Order Finite Elements for Convected Wave Propagation. International Journal for Numerical Methods in Engineering 96 (2013) 665-688.

[28] H. Bériot, A. Prinn, G. Gabard: Efficient Implementation of High-Order Finite Elements for Helmholtz Problems. International Journal for Numerical Methods in Engineering (2015) 1-47.

[29] P. Šolín, K. Segeth, I. Doležel: Higher-Order Finite Element Methods. CRC Press, Boca Raton, FL, 2004.

[30] A. Lieu, G. Gabard, H. Bériot: A Performance Study of High-Order Finite Elements and Wave-Based Discontinuous Galerkin Methods for a Convected Helmholtz Problem. 22 ${ }^{\text {nd }}$ International Congress on Sound and Vibration ICSV22, Florence, 2015.

[31] K. Hamiche, G. Gabard, H. Bériot: A Stabilised HighOrder Finite Element Model for the Linearised Euler Equations. 21st AIAA/CEAS Aeroacoustics Conference, Dallas, 2015.

[32] L. P. Franca, G. Hauke, A. Masud: Revisiting Stabilized Finite Element Methods for the AdvectiveDiffusive Equation. Computer Methods in Applied Mechanics and Engineering 195 (2006) 1560-1572.

[33] G. L. Beau, S. Ray, S. Aliabadi, T. Tezduyar: SUPG Finite Element Computation of Compressible Flows with the Entropy and Conservation Variables Formulations. Computer Methods in Applied Mechanics and Engineering 104 (1993) 397-422.

[34] J.-P. Bérenger: A Perfectly Matched Layer for the Absorption of Electromagnetic Waves. Journal of Computational Physics 114 (1994) 184-200.
[35] A. Bermúdez, L. Hervella-Nieto, A. Prieto, R. Rodríguez: An Optimal Perfectly Matched Layer with Unbounded Absorbing Function for Time-Harmonic Acoustic Scattering Problems. Journal of Computational Physics 223 (2007) 469-488.

[36] E. Bécache and A.-S. Bonnet-Ben Dhia and G. Legendre: Perfectly Matched Layers for the Convected Helmholtz Equation. SIAM Journal on Numerical Analysis 42 (2004) 409-433.

[37] R. M. Munt: The Interaction of Sound with a Subsonic Jet Issuing from a Semi-Infinite Cylindrical Pipe. Journal of Fluid Mechanics 83 (1977) 609-640.

[38] G. Gabard, R. J. Astley: Theoretical Model for Sound Radiation from Annular Jet Pipe: Far- and NearField Solutions. Journal of Fluid Mechanics 549 (2006) 315-341. 